\title{
RAZÓN Y SER DE LA FÁBRICA ARMADA
}

\section{(REASON AND BEING OF THE REINFORCED MASONRY)}

\section{Josep M. Adell Argilés, Dr. Arquitecto.}

Profesor Titular del Departamento de Construcción y Tecnología Arquitectónicas

Escuela T.S. de Arquitectura. Universidad Politécnica de Madrid

Miembro del Subcomité español CTN 140/SC6 "Estructuras de fábrica"

ESPAÑA

\section{RESUMEN}

Se analizan las razones arquitectónicas que impulsan la necesidad de considerar al acero como un nuevo componente de las fábricas.

Se exponen las posibles maneras de conseguir dicha combinación siguiendo el ser esencialmente constructivo de la albañilería.

La fábrica resultante de ello es un nuevo material compuesto, de carácter arquitectónico, que puede constituirse en tipología estructural.

\section{SUMMARY}

This article is an analysis of the reasons why steel can be seen as a new component of masonry.

It explains the waps in which steel can be effectivily used in masonry.

The combination of steel and masonry can be regarded as a new composite material, which can extend architeturas bounderies through its structural use.

“Cada material tiene una personalidad específica y distinta, y cada forma impone un diferente fenómeno tensional. La solución natural de un problema - arte sin artificio-, óptima frente al conjunto de impuestos previos que la originan, impresiona con su mensaje, satisfaciendo, al mismo tiempo, las exigencias del técnico y del artista.

El nacimiento de un conjunto estructural, resultado de un proceso creador, fusión de técnica con arte, de ingenio con estudio, de imaginación con sensibilidad, escapa del puro dominio de la lógica para entrar en las secretas fronteras de la inspiración. Antes y por encima de todo cálculo está la idea, moldeadora del material en forma resistente, para cumplir su misión".

A esa idea, va dedicado el libro “Razón y ser de los tipos estructurales”, escrito por Eduardo Torroja (1).

\section{RAZÓN ARQUITECTÓNICA}

El acero en el siglo XX ha revolucionado la Arquitectura, con su capacidad a tracción. Como constitutivo de las estructuras de perfiles laminados o de hormigón armado ha permitido el trabajo a flexión de los elementos estructurales. La viga ha sustituido al arco.
Las fábricas se caracterizan por una buena capacidad de trabajo a compresión frente a una despreciable a tracción.

El arco, gracias a su organización constructiva, permite salvar el vano, obligando a un recorrido curvo de los esfuerzos a compresión, lo que se traduce en empujes sobre los apoyos, función de su peralte. 
El ritmo vertical de vacíos y macizos, de arcos y estribos, ha presidido la composición de las fábricas durante siglos.

El empuje del arco puede evitarse, si es absorbido por la tracción del acero de un tirante. El conjunto "arcotirante" transmite solamente cargas verticales, permitiendo diseñar la arquitectura de fábrica con apoyos de más esbeltas proporciones.

Para evitar los problemas de corrosión del acero se aconseja un tratamiento eficaz. El hormigón cumple perfectamente esta labor envolviendo las armaduras.

Louis Kahn, el Arquitecto norteamericano, expresó el camino hacia la fábrica armada, con originales planteamientos de gran riqueza arquitectónica, combinando el acero y la fábrica (Fig. 1).

Se tiene la necesidad de lograr una estudiada combinación de los materiales de fábrica con el acero, que permita actualizar el comportamiento de las fábricas de acuerdo con las técnicas de construcción de nuestro tiempo. El objetivo está en solventar la problemática sobre la fisuración que presentan las fábricas, potenciando a la vez sus posibilidades arquitectónicas.

Veamos a continuación algunas de las razones que apoyan esta idea.

En el siglo XIX, el ladrillo se consideró como una pieza modular fabricada contando con el ancho de las juntas, lo que regularizó la construcción de las fábricas. El aparejo con su variada pero estricta ordenación, constituye un primer paso hacia la modernidad. Los muros que eran lo suficientemente gruesos y de organización homogénea podían cumplir a la vez todas las funciones requeridas (2).

En nuestro siglo, por cuestiones económicas y de eficiencia, el muro se ha dividido en hojas. El muro capuchino, o "cavity wall", se ha impuesto como la solución más idónea pera la mayoría de las fábricas.

Es habitual separar las funciones "resistente" y de "acondicionamiento" que tradicionalmente cumplían las fábricas de gruesos muros por sí mismas, y resolver la estructura con reticulados de acero u hormigón armado, quedando las fábricas con una función especializa- da de cerramiento. Además esto permite mayor libertad en la composición de los huecos que pueden variar su ritmo vertical por la horizontalidad.

Los materiales que hoy día se fabrican para la albañilería se han especializado en alguno de los cometidos que han de cumplir en la fábrica.

Si dejamos al margen los materiales pétreos, que han pasado a utilizarse casi exclusivamente como chapados, podemos destacar:

- Los materiales cerámicos que suman a sus valores de tradición y cualidades higrotérmicas, los de elevada resistencia. Los hay poco pesados, como los bloques cerámicos aligerados.

- Los conglomerados de hormigón, que destacan por sus grandes piezas huecas que pueden armarse verticalmente, permitiendo gran variedad de colorido y tratamientos de acabado.

- Los morteros celulares, que añaden a su gran ligereza y tamaño un magnífico comportamiento térmico frente al fuego, con muy buen acabado y fácil trabajabilidad.

Por los avances experimentados en la construcción de obras de fábrica, se prevé un aumento en la aplicación de la albañilería en los próximos años (3).

Por razones de economía, en material, espacio, mano de obra etc... las fábricas toman el mínimo grueso posible: el ancho de las piezas. Éstas se fabrican del máximo tamaño que permita su manejabilidad, lo que lleva a construir muros de fábrica de gran esbeltez.

La esbeltez de las fábricas compromete su estabilidad frente a acciones diversas, debiendo de recurrir al atado de hojas, anclajes a soportes, forjados, etc. Este hecho da lugar a conexiones entre la estructura y el cerramiento que interfieren a la actuación libre de ambos. Por consiguiente, la supuesta separación de funciones de hecho es más teórica que real en la práctica, especialmente a lo largo del paso del tiempo por efectos diferenciales en los movimientos y asentamientos de la estructura y del cerramiento.

Las estructuras de acero y de hormigón arrastran en sus deformaciones a las fábricas, caracterizadas por poseer materiales de gran rigidez, produciendo en ellos la fisu- 


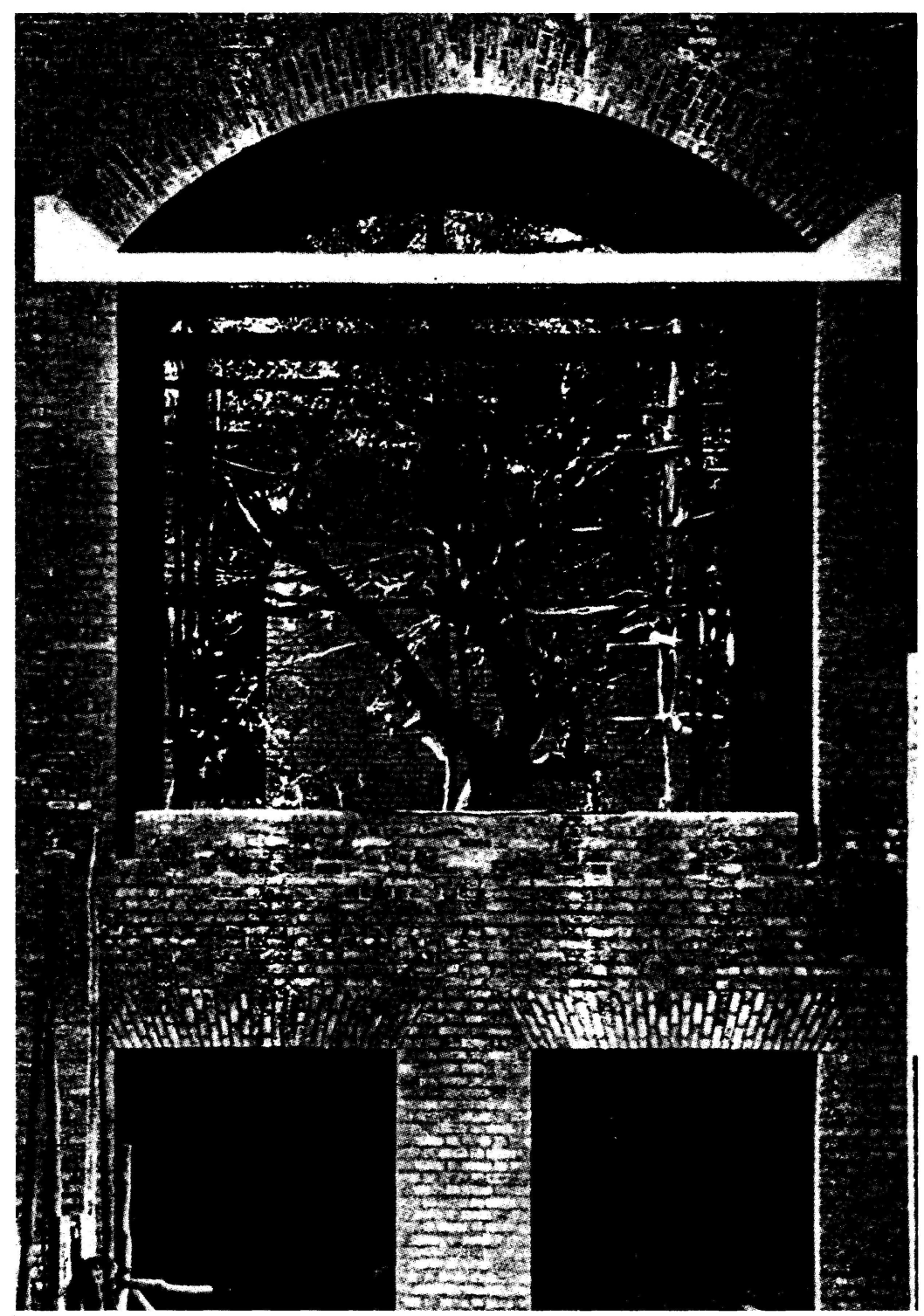

Fig. 1.-Arco rebajado de fábrica de ladrillo. Tirante de hormigón armado. (Louis Kahn. Instituto Hindú de Cuadros. Ahmedabad 1963).

ración. Se acusa una patología general de amplio espectro, que no se manifestaría en el caso de que las fábricas tuvieran capacidad a tracción.

La calidad de vida en Europa hoy día no admite la aparición de fisuras en las fábricas. Las Constructoras, Casas de Seguros, la Administración, etc. necesitan poder garantizar que no se produzca esta patología. En muchos países, como Bélgica, Holanda, Suiza, etc., esto es objeto de especial atención, existiendo una amplia experiencia en el conocimiento de las causas que generan la fisuración de las fábricas y en cómo evitarlas con el armado (4).

Si se consideran las funciones que cumplen los materiales de fábrica en una edificación analizando sus cualida- des, pronto se comprueba que difícilmente pueden sustituirse por materiales de otra índole, dado el alto grado de idoneidad a que han llegado.

Sin embargo, la masa que estos materiales aportan a la edificación podría aprovecharse con capacidad estructural, dadas sus cualidades resistentes, colaborando en soportar el edificio, lo que en vez de ser una carga añadida a la estructura se convertiría en un ahorro de ésta.

Aprovechar el material de albañilería del cerramiento, medianeras, caja de escaleras, etc. para que actue además como fábrica resistente, supone un importante ahorro en el coste de la obra e incide en el ahorro energético global del proceso de edificación. 
Informes de la Construcción, Vol. 44 n. ${ }^{\circ}$ 421, septiembre/octubre, 1992

Dieste lo expresa con el concepto de "economía cósmica", entendido como el aprovechamiento óptimo de las cualidades de la materia. Véase la estructura cerámica en "Las bóvedas de la Atlántida" (5).

Para aumentar la capacidad estructural de las delgadas fábricas de albañilería sin aumentar su grueso, es necesario estudiar una forma de armado que lo haga posible. Ésta ha de ser válida para las diversas obras de fábrica, y por supuesto también para las que cumplen un papel estático-resistente.

Otra causa importante que obliga a introducir el acero en las fábricas es el sismo. En España hay varias zonas de grado sísmico elevado, donde se plantea el problema de la necesidad de tener que construir obras de fábrica dúctiles. La normativa sobre el tema exige armar la fábrica en estos casos.

La experiencia sísmica italiana nos es de gran ayuda, habiendo sido reflejada por el profesor Calvi en el artículo "Cálculo de estructuras de fábrica armada contra el sismo" (6).

Junto a estas razones que suscitan la necesidad de armar las fábricas, no podemos olvidar los valores de carácter histórico, estético, del entorno, etc. que suelen ser muy decisivas a la hora de escoger determinados materiales de la albañilería.

Incluso es habitual aplicarla en contraste con los muros cortina de vidrio.

\section{SER CONSTRUCTIVO}

Analizadas algunas de las razones que aconsejan armar la fábrica, queda encontrar el cómo hacerlo.

La consideración genérica de "la fábrica", que contempla en una sola expresión varios tipos de materiales distintos, organizados constructivamente, obliga a la búsqueda de una "forma generalizada de armar la fábrica" que se acomode a las reglas de ejecución de la albañilería.

De poco sirven las pautas del hormigón armado cuando éste se trata de un líquido que se vierte dentro de un encofrado donde previamente se ha dispuesto la ferralla, organizando los redondos con libertad direccional y dimensional.
Por el contrario, la obra de fábrica, que tiene la ventaja de no requerir encofrado, se levanta por niveles, colocando las piezas en discontinuidad vertical de llagas, sentadas en los tendeles horizontales de la fábrica.

A la hora de "armar la fábrica", habrá que contemplar pues la "técnica de la albañilería", para encontrar las opciones que la fábrica ofrece para ello.

A pesar de que existen materiales de fábrica diversos que pueden dar lugar a fábricas con distintas combinaciones y aparejos, permanece como constante la ejecución por niveles de la albañilería, haciéndose los tendeles con escaso grueso de mortero.

Para poder armar "cualquier tipo de fábrica", habrá que aprovechar "los tendeles" como "invariantes" que son de la albañilería, ya que la mayoría de los materiales no disponen de huecos lo suficientemente amplios para poder introducir armaduras por ellos.

El "escaso grueso del tendel", de alrededor de $1 \mathrm{~cm}$, y la necesidad de un suficiente recubrimiento de mortero envolviendo la armadura, "obliga a armar con alambres", en vez de con redondos.

Sin embargo, "armar con alambres" sin más, tiene "enormes dificultades" desde un punto de vista práctico:

- la dificultad de manejar los alambres sueltos por su tendencia a enrollarse,

- la dificultad de colocarlos en el tendel en la posición precisa para poder cumplir su cometido estructural, puesto que se desplazan al colocar las piezas.

Todo ello hace necesario diseñar una "organización prefabricada de alambres" que mantenga una forma estable, asegure una adecuada colocación en la fábrica y, además, permita no entorpecer el normal proceso de ejecución de la albañilería al armarla.

Una vez desarrollado un sistema eficaz de armar la fábrica, éste deberá ir acompañado de toda una investigación en laboratorio y en obra que lo refrende, así como de un Manual que dé a conocer la adecuada aplicación de la nueva técnica.

La industria que se ha ocupado de esta problemática, elabora "armaduras prefabricadas planas para tendeles" de manera idónea. Existen básicamente dos tipos distin- 
tos de éstas: la que se utiliza en América desde hace cincuenta años, y la versión más desarrollada de Europa, con dos décadas de experiencia.

La armadura europea Murfor fabricada por la casa Belga BEKAERT, aventaja a la americana en la forma de mantener paralelamente unidos los dos alambres principales mediante un alambre diagonal en zig-zag, dispuesto en el mismo plano de los longitudinales (Fig. 2).

El grueso del conjunto es de solamente los 4 ó $5 \mathrm{~mm}$ de diámetro del alambre principal (existe la variante de pletina con sólo $2 \mathrm{~mm}$ de grueso, para juntas de mortero cola). Su estructura, al estar triangulada, es indeformable. La triangulación aumenta la adherencia de la armadura prefabricada con el mortero del tendel y absorbe las tensiones de rasante entre los paramentos interior y exterior de la fábrica por diferencias térmicas y/o acciones horizontales. Al tratarse de un alambre contínuo, no presenta puntos de discontinuidad propensos a la corrosión, suponiendo además un $40 \%$ extra de acero a favor de la seguridad.

Para poder satisfacer el amplio campo de aplicación de las fábricas armadas, es necesario prefabricar las armaduras planas con una amplia gama de separaciones estándar, puesto que existen materiales de fábrica diversos, cada cual con su proporción y tamaño. Las fábricas se pueden organizar combinándolas entre sí, en razón de las cualidades a exigir al conjunto de las hojas de fábrica: estabilidad, resistencia, aislamiento, estética, etc.

Es importante resaltar que, con estas armaduras prefabricadas, no sólo se puede armar cada muro de fábrica, sino atar entre sí diversas hojas, permitiendo muros doblados o capuchinos de mayor inercia con fábricas de distintas características. Su uso supone gran facilidad de ejecución con ahorro de tiempo y coste (Fig. 3).

Los campos de aplicación de la fábrica armada, así como los consejos para su colocación, que es algo bien conocido en otros países, requiere que se dé una adecuación a la normativa y a las circunstancias de cada lugar.

El que suscribe ha elaborado el Manual Murfor sobre la fábrica armada para BEKAERT, recogiendo las particularidades de nuestro país para dar a conocer las grandes posibilidades de esta nueva técnica (7).

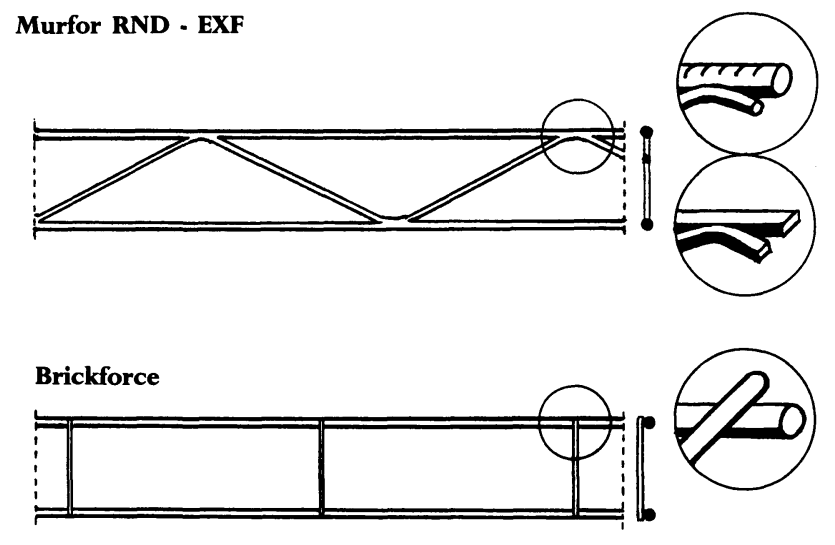

Fig. 2.-Armaduras prefabricadas planas para la fábrica armada.

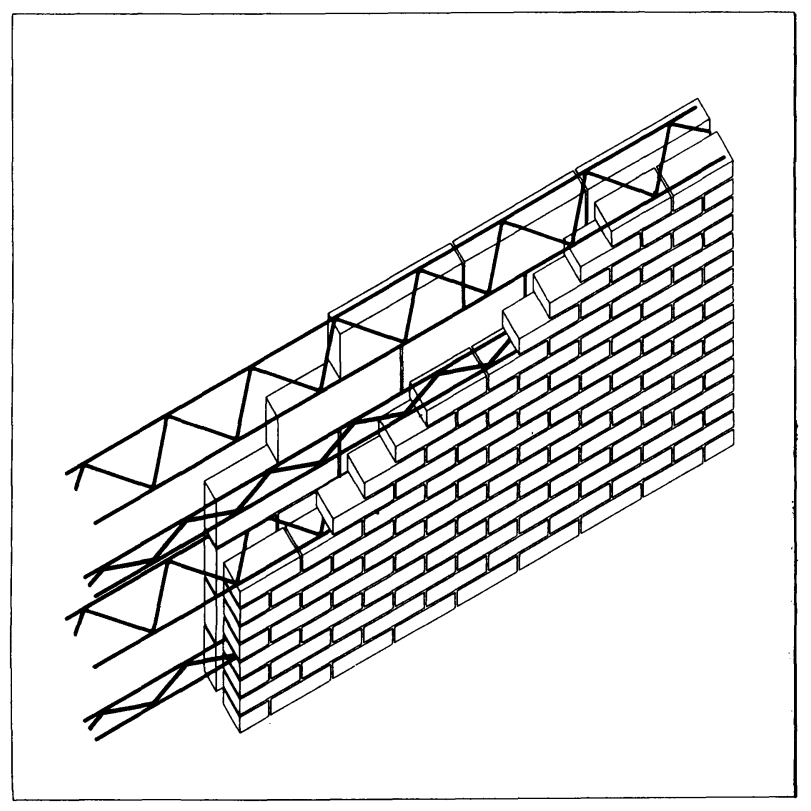

Fig. 3.-Muro capuchino de fábrica armada, con hojas de distinto material de fábrica. 
La disposición horizontal de armaduras en los tendeles no puede resolver todos los planteamientos estructurales de las fábricas. Recientemente se ha desarrollado una técnica siguiendo el espíritu de la albañilería para armar también verticalmente.

Se han diseñado "armaduras prefabricadas de carácter espacial" o "tridimensionales", que "permiten armar bidireccionalmente" algunas fábricas aprovechando la existencia de materiales que tienen amplios huecos, como los bloques de hormigón. También se ha pensado en otros que puedan desarrollarse, como los bloques cerámicos aligerados y con huecos, que ya se fabrican en Suiza.

La patente Murfor RE, en honor al Ingeniero suizo Reinle que la ideó, permite armar horizontal y verticalmente a la vez la fábrica, siguiendo el proceso de ejecución de la albañilería (Fig. 4).

Este nuevo sistema, que simultáneamente se está introduciendo en varios países de Europa, supondrá por sus prestaciones una revolución técnica de las fábricas, ya que las pone en competencia frente al hormigón armado al permitir que las fábricas sean sometidas a solicitaciones de compresión y flexión compuesta.

Además ofrece las ventajas de las cualidades de los materiales de albañilería construidos con una sencilla técnica de ejecución, y sin los costes del encofrado ni la dependencia del hormigonado.

En la Universidad de Lieja se han ensayado estas armaduras modulares con cercos, ofreciendo resultados más satisfactorios que los correspondientes al armado de los bloques huecos de hormigón, con equivalente armado convencional de redondos (8).

Una vez planteada de forma genérica lo que constituye "el ser" de la fábrica armada, conviene afrontarlo con toda seriedad para poder asegurar que no habrá problemas de corrosión.

Es conocido que las armaduras, si bien quedan envueltas con mortero de cemento que las protege de la corrosión, éste no tiene las mismas cualidades que un buen hormigón. La evidencia de este hecho obliga a utilizar para la fábrica armada solamente armaduras prefabricadas con un tratamiento suficientemente garantizado.
Realizar ocasionalmente en obra algún refuerzo de fábrica con redondos, que apenas tendrán recubrimiento de mortero dado su excesivo grueso frente al del tendel y sin tratamiento alguno, es una temeridad que hay que eludir porque termina provocando la corrosión con las consiguientes consecuencias negativas.

La experiencia belga con armaduras Murfor, suficientemente contrastada en laboratorio y en la realidad, aconseja armaduras galvanizadas en paredes interiores, galvanizadas más un recubrimiento de capa epoxi para exteriores, e incluso de acero inoxidable para ambientes industriales o muy agresivos. Véase el artículo de Pfeffermann y Haseltine "El desarrollo de armaduras para tendeles a lo largo de dos décadas" (9).

Aparte del armado por tendeles que constituye la "fábrica armada", por cuanto es válido para cualquier material de albañilería, existen otras formas de armar las fábricas localizadamente, las cuales consisten en adaptar las piezas a las pautas del armado. Estas otras formas deben de entenderse más bien como maneras de "reforzar la fábrica", por cuanto no constituyen un armado homogéneo de las mismas. No obstante, en determinadas situaciones, pueden ser de gran interés en combinación con la fábrica armada (10).

\section{TIPO ESTRUCTURAL}

Considerar a la fábrica armada como un "tipo estructural" nuevo quizá sea algo excesivo, ya que participa de las cualidades de otros tipos claramente diferenciados, y porque muchas veces colabora con otros sin desempeñar un papel independiente.

No obstante, es evidente que los conceptos genéricos de "la fábrica" y "fábrica armada", tal y como se han expuesto, tienen importantes diferencias en su forma de comportarse gracias a la colocación de armaduras prefabricadas en los tendeles.

La fábrica armada añade a las cualidades propias del material de fábrica utilizado la capacidad de soportar al mismo tiempo: "deformaciones impuestas", "flexiones verticales" y "flexiones horizontales".

Analicemos sencilla y separadamente estos conceptos: (Fig. 5). 
(A) "Fábrica sometida a deformaciones impuestas"

Las causas comunes que generan deformaciones impuestas son de orden térmico como la DILATACIÓN o la CONTRACCIÓN, o bien por motivos reológicos como la RETRACCIÓN. Esto obliga a hacer juntas de dilatación que eviten la fisuración del material de fábrica a distancias regulares máximas dependiendo del material.

En una fábrica homogéneamente armada, es decir, con armaduras regularmente dispuestas en los tendeles de "ancho (a)" y "alto (h)", se puede aumentar sensiblemente la "longitud (l)" de separación de juntas en los paños de fábrica. Este aumento de la longitud es función de la separación vertical de los tendeles armados y del tipo de material de fábrica utilizado.

Para que esta armadura considerada secundaria sea eficaz debe de contemplar un mínimo del $0,05 \%$ de la sección del muro.

\section{(B) "Fábrica sometida a flexión vertical"}

Como los materiales de albañilería son incapaces de soportar las tensiones que se producen en la zona traccionada de una fábrica sometida a flexión vertical, será preciso disponer en ella la cuantía de armado precisa a tracción como armado principal.

La "acción" que será capaz de soportar una fábrica de "ancho (a)" sometida a flexión vertical entre apoyos a una "luz (l)" concreta, dependerá de las posibilidades que haya de ubicar las armaduras de manera localizada a una "altura efectiva (he)".

Esta altura efectiva estará en función de la separación vertical de los tendeles, que depende de la altura de las piezas y del grueso del muro y ancho de las armaduras prefabricadas usadas.

Visto de otra forma, para una acción conocida como puede ser la carga de un DINTEL, o el peso de un MURO DIVISORIO sometido a efecto arco por descenso de la base de apoyo (comúnmente el forjado) de una fábrica de "ancho (b)" y alto (h), se podrá aumentar la "luz (l)" del vano entre apoyos en función del armado que se disponga en los tendeles inferiores, siempre que haya suficiente canto eficaz (he).
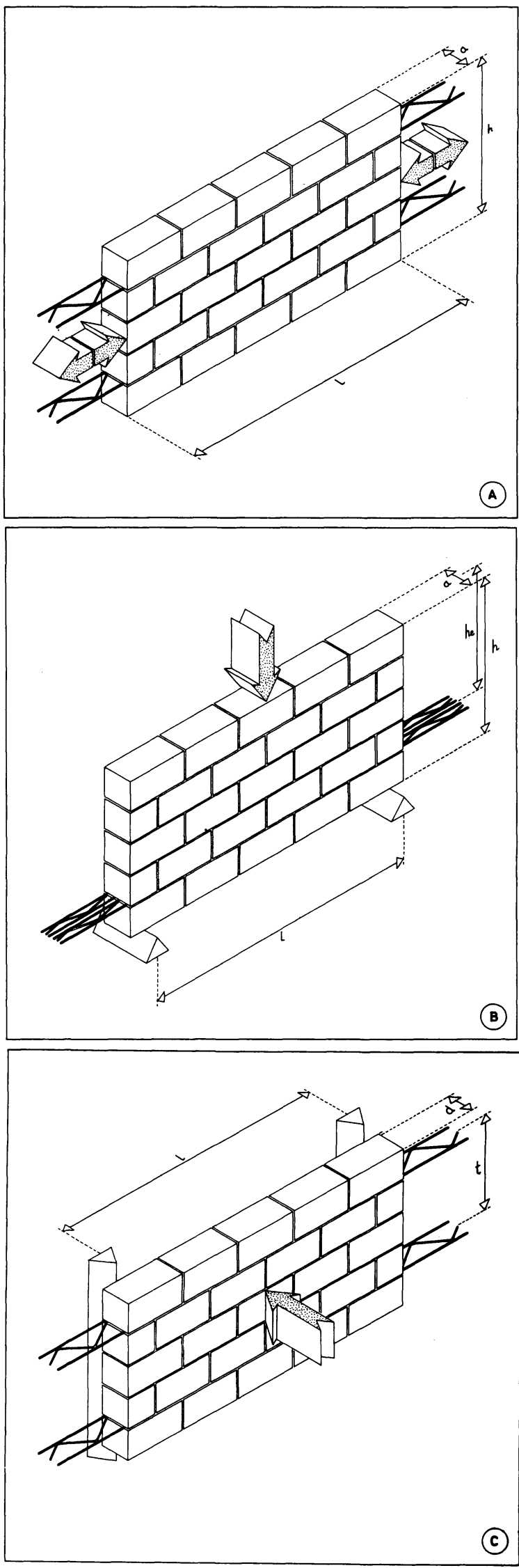

Fig. 5.-Fábrica armada sometida a diversas solicitaciones:

(A) Fábrica sometida a deformaciones impuestas.

(B) Fábrica sometida a flexión vertical.

(C) Fábrica sometida a flexión horizontal. 
Informes de la Construcción, Vol. 44 n. ${ }^{\circ}$ 421, septiembre/octubre, 1992

\section{(C) "Fábrica sometida a flexión horizontal"}

Una fábrica sometida a flexión horizontal entre apoyos verticales a determinada "luz (l)", con una "distancia (d)" entre la zona traccionada y comprimida de su sección horizontal, podrá soportar una ACCIÓN EÓLICA, EMPUJE DEL TERRENO, etc., determinada en función de la regular separación en altura a que estén dispuestos los tendeles armados.

Es decir, aumentando la cantidad de tendeles armados, podrá aumentarse la luz entre apoyos para un determinado material de fábrica de ancho dado, lo que supone economizar en soportes, costillas, retallos, etc.

O por el contrario, podrá economizarse en el grueso del material de fábrica a utilizar si se mantiene la luz entre apoyos al disponer más tendeles armados.

En todos los casos (A), (B) y (C), según el material utilizado, influye decisivamente la capacidad de soportar compresiones que tenga la fábrica, así como su resistencia al corte.

En la fábrica armada existe una componente de la compresión, que sigue la dirección de los tendeles, que puede obtenerse experimentalmente. De su efectividad depende el correcto rejuntado de las llagas con mortero. Por otra parte también es decisivo el rasante entre las piezas de la fábrica y el mortero.

Los supuestos analizados teóricamente han sido ensayados con muretes escala 1:1 en laboratorio, a fin de comprobar el comportamiento real de los diversos materiales de fábrica conjuntamente con el acero de las armaduras Murfor gracias a la acción compuesta ejercida por el mortero. Ello ha permitido poder establecer una correlación entre la teoría y la práctica que garantice la adecuada generalización de la fábrica armada. Estos ensayos se recogen por Pfeffermann y Baty en "Maçonnerie armee" (11).

Una vez tipificado el acero, y después de fijar las cualidades resistentes mínimas que han de cumplir el mortero y los materiales, se han ensayado los diversos tipos de fábrica. Con ello se han podido determinar las longitudes de anclaje entre armaduras, los recubrimientos mínimos de mortero, etc. pudiéndose fijar las reglas que permiten garantizar el funcionamiento del sistema en base a estas armaduras específicas.
Los principios de cálculo de la fábrica armada, así como la elaboración de tablas de cálculo que simplifican los casos más habituales, han sido preparados por el profesor Lahuerta y recogidos en el Manual Murfor de BEKAERT, exponiéndose en su artículo (12).

El cálculo de la fábrica armada toma como referencia el del hormigón armado. La versión española del manual aporta un sustancial matiz respecto a otros países, ya que el técnico puede aceptar la fisuración controlada debido a cargas permanentes a la hora de calcular dinteles de fábrica armada bajo la carga de forjados.

Las ventajas que reporta la combinación del acero y la cerámica han sido analizadas en el libro "El muro de ladrillo" de Hispalyt, con el título "Los materiales cerámicos y la fábrica armada" (13).

\section{NUEVO MATERIAL COMPUESTO ARQUITECTÓNICO}

Las tres formas básicas de trabajar de una fábrica armada por tendeles, se han expresado por separado en (A), (B) y (C) para facilitar la exposición (Fig. 5).

En la realidad es habitual que se superpongan varias de estas acciones o inclusive todas ellas en una misma fábrica, lo que se afronta perfectamente sin más que disponer la cuantía de armado precisa y en la ubicación correcta, según las solicitaciones existentes.

Sin embargo, para poder ajustarse lo más posible a la cuantía de armado requerida, puede ser preciso utilizar varios anchos de armaduras prefabricadas, con el fin de que quepan más de una en un tendel (flexión vertical), o bien para garantizar la correcta posición del alambre traccionado con el máximo brazo que permita el grueso de la fábrica (flexión horizontal).

De todo ello se deduce que, al igual que en el hormigón armado, en la fábrica armada es posible dosificar (ajustar) la cuantía del armado y su disposición, si bien está limitado por la separación y anchura de los tendeles y en función de la altura y anchura de las piezas.

El borrador de la nueva normativa europea EC6, contempla la cuantía mínima de armado que ha de tener una fábrica para considerarse "fábrica armada", cifrándolo en el mínimo del $0,05 \%$ de la sección de la fábri$\mathrm{ca}$, que ha de distribuirse uniformemente. Esto equivale, 
según el grueso de fábrica, a disponer armaduras cada 40 ó $60 \mathrm{~cm}$ de altura (14).

Si se construye la fábrica siguiendo los anteriores consejos, el producto obtenido puede considerarse como un nuevo material compuesto. Sus cualidades y características se corresponden con las del material de las piezas, soportando el acero la tracción de las acciones previamente calculadas evitando al tiempo la fisuración. El mortero se encarga de ejercer la acción compuesta entre la matriz y la fibra.

El nuevo material compuesto "fábrica armada" es dúctil en vez de frágil y tiene un comportamiento homogéneo, puesto que además tiene el armado mínimo regularmente repartido que impide la rotura frágil sin presentar, por armarse, heterogeneidad con perfiles o zunchos de hormigón.

El Arquitecto, Ingeniero, etc. tienen la posibilidad de "crear" el material compuesto "fábrica armada" con la proporción de armado necesaria y su colocación precisa, de acuerdo a criterios de diseño propios en beneficio de la construcción arquitectónica.

Las fábricas así diseñadas, podrán relacionarse con hojas de otras características, soportar cargas de forjados, acciones de viento, empujes de terrenos, atar encuentros de muros..., incluso constituirse en la estructura del edificio, eludiendo los puentes térmicos.

La fábrica armada viene a ocupar un vacío existente entre los materiales de construcción, a mitad de camino entre la obra de fábrica tradicional y el hormigón armado.

\section{"LA FÁBRICA ARMADA"}

La fábrica armada por tendeles debe entenderse como: "UN NUEVO MATERIAL COMPUESTO ARQUITECTÓNICO", que permite potenciar la Arquitectura tanto desde el punto de vista de la técnica como de la forma.

Esta manera de entender la fábrica armada y sus posibilidades fue expuesta en la I Jornada sobre aplicaciones arquitectónicas de los materiales compuestos y aditivados (15).

Las ventajas que el armado reporta a las fábricas son evidentes; el enriquecimiento que puede obtener la arquitectura con una sabia revitalización de las fábricas está todavía en sus inicios. Algunas de las primeras realizaciones que se están llevando a cabo se presentan en el artículo "Arquitectura e Investigación con fábrica armada" (16).

Como ya comentó el autor en la conferencia expuesta en los "Seminarios Torroja", bajo el título "De la Arquitectura de Ladrillos a la fábrica armada" (17).

En la evolutiva mejora de las fábricas, a través de los tiempos: se pasó de "la trabazón" "al aparejo" y éste da paso al "armado".

En la fábrica armada, el dintel sustituye formalmente al arco como corresponde a la modernidad de la técnica, pero el material internamente trabaja a compresión, siguiendo una tendida directriz arqueada de esfuerzos equilibrados por la tracción del acero que actúa de tirante eludiendo los empujes (Fig. 6).

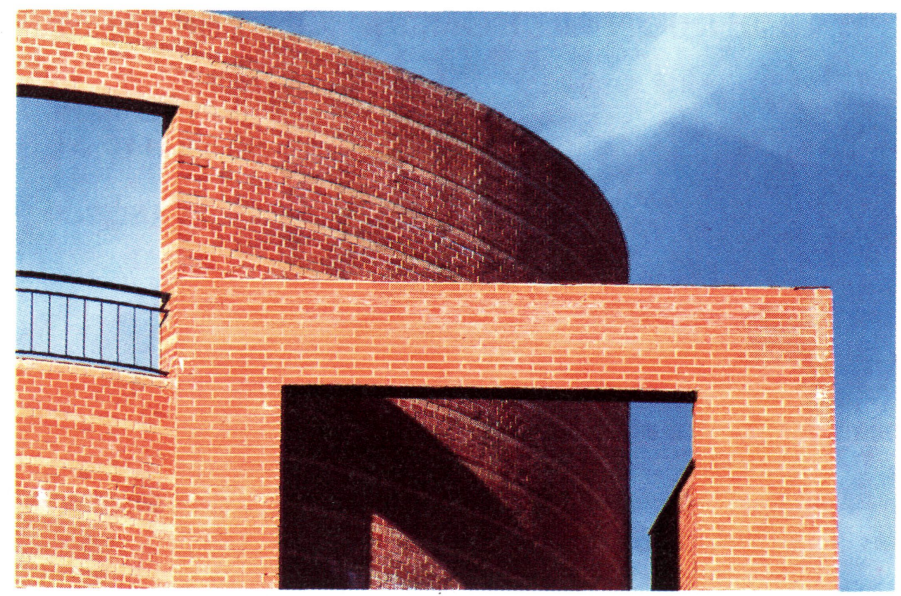

El arco existe aunque no se evidencie:

"la fábrica tiene vida".

Fig. 6.-Arco rebajado de fábrica armada.

(Josep M. Adell. Viviendas en la Plaza de la Remonta. Madrid 1991). 
Informes de la Construcción, Vol. 44 n. ${ }^{\circ}$ 421, septiembre/octubre, 1992

BIBLIOGRAFÍA

(1) Eduardo Torroja. Razón y ser de los tipos estructurales. Textos universitarios. Consejo S. de Investig. Científicas. Madrid 1 . $^{\text {a }}$ edición 1957, 7. edición 1991.

(2) Josep M.a Adell. La Arquitectura de ladrillos del siglo XIX: Racionalidad y modernidad.

Arquitectura de ladrillos del siglo XIX. Técnica y forma. Libro: Fundación Universidad-Empresa, Madrid 1985. Artículo: Informes de la Construcción n. 421 , Instituto Eduardo Torroja. Madrid. Consejo Sup. Invest. Científ. Septiembre-octubre 1992.

(3) Walter Mann. Avances en la construcción de obras de fábrica. Informes de la Construcción n. 421 , Instituto Eduardo Torroja. Madrid. Consejo Sup. Invest. Científ. Septiembre-octubre 1992.

(4) Jan Stasse. La postura holandesa frente al control de la fisuración. Informes de la Construcción n. 421 , Instituto Eduardo Torroja. Madrid. Consejo Sup. Invest. Científ. Septiembre-octubre 1992.

(5) Josep M. Adell. Las bóvedas de la Atlántida. Informes de la Construcción n. ${ }^{0} 421$, Instituto Eduardo Torroja. Madrid. Consejo Sup. Invest. Científ. Septiembre-octubre 1992.

(6) Michele Calvi. Cálculo de estructuras de fábrica armada contra el sismo.

Informes de la Construcción n. 421 , Instituto Eduardo Torroja. Madrid. Consejo Sup. Invest. Científ. Septiembre-octubre 1992.

(7) Josep M. A Adell. La fábrica armada. Manual Murfor de Bekaert. Bekaert Ibérica, S. A. Barcelona 1992.

(8) Luiz Oliveira. La armadura tridimensional para la fábrica armada. Informes de la Construcción n. 421 , Instituto Eduardo Torroja. Madrid. Consejo Sup. Invest. Científ. Septiembre-octubre 1992.
(9) Oscar Pfeffermann / Barry Haseltine. El desarrollo de armaduras para tendeles a lo largo de dos décadas.

Informes de la Construcción n. 421 , Instituto Eduardo Torroja. Madrid. Consejo Sup. Invest. Científ. Septiembre-octubre 1992.

(10) Berstein / Champetier / Peiffer. Nuevas técnicas en la obra de fábrica.

Edt. Gustavo Gili, Barcelona 1985. (1.a edición París 1982).

(11) Pfeffermann / Baty. Maçonnerie Armee.

Centre Scientifique et Techique de la Construction. № 26 Compe rendu d'étude et de recherche Bruselas 1981.

(12) Javier A. Lahuerta. Cálculo de la fábrica armada. Informes de la Construcción n. 421, Instituto Eduardo Torroja. Madrid. Consejo Sup. Invest. Científ. Septiembre-octubre 1992.

(13) Josep M.a Adell. Los materiales cerámicos y la fábrica armada. El muro de ladrillo. Hispalyt. Madrid 1992.

(14) Eurocode EC6. Part 2. Reinforced masonry structures. Commision of the European Communities, Bruselas 1989.

(15) Josep M. Adell. La fábrica armada un nuevo material arquitectónico. Aplicaciones arquitectónicas de los materiales compuestos y aditivados. 1. Jornada 13-XII-91. Departamento de Construcción y Tecnologías Arquitectónicas de la Escuela $T$. S. de Arquitectura de la U.P. de Madrid.

(16) Josep M. Adell. Arquitectura e Investigación con fábrica armada. Informes de la Construcción n. 421, Instituto Eduardo Torroja. Madrid. Consejo Sup. Invest. Científ. Septiembre-octubre 1992.

(17) Josep Ma. Adell. De la Arquitectura de ladrillos a la fábrica armada. Seminarios Torroja, conferencia 7-XI-91. Informes de la Construcción n. 416, Instituto Eduardo Torroja. Madrid. Consejo Sup. Invest. Científ. Septiembre-octubre 1992.

\title{
Última monografía del ICCET/CSIC
}

\section{N. ${ }^{\circ} 409$}

\section{OCLUSIÓN DE POROS POR TRATAMIENTO QUÍMICO}

\author{
FERNANDO TRIVIÑO VAZZQUEZ \\ IGNACIO MENÉNDEZ DE PAZOS \\ ICCET/CSIC \\ FRANCISCO HERNÁNDEZ LÓPEZ \\ ETSICCPM
}

Madrid, junio 1992

Se usa el principio del producto de solubilidad para rellenar los poros de materiales de construcción, impregnándolos con dos sales que formen otra insoluble.

Se estudian, a diversas temperaturas, los materiales siguientes: morteros de cemento, ladrillos y escayolas, tratados con diversas sales cálcicas como impregnantes y oxalatos y carbonatos como precipitantes, obteniéndose de esta forma una ocupación de poros del orden del $70 \%$. 